\title{
AN IDENTITY FOR THE DEDEKIND ETA-FUNCTION INVOLVING TWO INDEPENDENT COMPLEX VARIABLES
}

\author{
BRUCE C. BERNDT ${ }^{1}$ and WILLIAM B. HART
}

\section{INTRODUCTION}

Recall that the Dedekind eta-function $\eta(\tau)$ is defined for $q=e^{2 \pi i \tau}$ and $\tau \in \mathcal{H}=\{\tau$ : $\operatorname{Im} \tau>0\}$ by

$$
\eta(\tau)=q^{1 / 24}(q ; q)_{\infty}
$$

where

$$
(a ; q)_{\infty}:=\prod_{n=0}^{\infty}\left(1-a q^{n}\right)
$$

The purpose of this paper is to prove the following striking identity for the etafunction, of which we know no other examples of a similar type.

Theorem 1.1. For $w, z \in \mathcal{H}$,

$$
\begin{aligned}
27 \eta^{3}(3 w) \eta^{3}(3 z)= & \eta^{3}\left(\frac{w}{3}\right) \eta^{3}\left(\frac{z}{3}\right) \\
& +i \eta^{3}\left(\frac{w+1}{3}\right) \eta^{3}\left(\frac{z+1}{3}\right)-\eta^{3}\left(\frac{w+2}{3}\right) \eta^{3}\left(\frac{z+2}{3}\right) .
\end{aligned}
$$

We describe now the genesis of (1.1). In preparing his doctoral thesis [2], the second author searched for modular equations involving

$$
u_{1}(\tau):=\frac{\eta(\tau / m)}{\eta(\tau)} \text { and } v_{1}(\tau):=u_{1}(n \tau),
$$

(and various modular transforms thereof). His goal was to generalize the modular equations of 'irrational kind' for the Weber functions

$$
\mathfrak{f}(\tau):=e^{-\pi i / 24} \frac{\eta\left(\frac{\tau+1}{2}\right)}{\eta(\tau)}, \quad \mathfrak{f}_{1}(\tau):=\frac{\eta\left(\frac{\tau}{2}\right)}{\eta(\tau)}, \quad \mathfrak{f}_{2}(\tau):=\sqrt{2} \frac{\eta(2 \tau)}{\eta(\tau)},
$$

discussed in $\S 75$ of Weber's book [3], i.e., the case $m=2$ in (1.2). For example, if $n=3$, letting

$$
u(\tau):=\mathfrak{f}(\tau), \quad u_{1}(\tau):=\mathfrak{f}_{1}(\tau), \quad u_{2}(\tau):=\mathfrak{f}_{2}(\tau)
$$

and

$$
v(\tau)=\mathfrak{f}(3 \tau), \quad v_{1}(\tau):=\mathfrak{f}_{1}(3 \tau), \quad v_{2}(\tau):=\mathfrak{f}_{2}(3 \tau),
$$

\footnotetext{
${ }^{1}$ Research partially supported by grant MDA904-00-1-0015 from the National Security Agency.
} 
one can prove the identity

$$
u^{2} v^{2}=u_{1}^{2} v_{1}^{2}+u_{2}^{2} v_{2}^{2}, \quad \tau \in \mathcal{H} .
$$

Generally, Weber's modular equations depend on $n$ and increase in complexity as $n$ increases.

In attempting to generalize these modular equations, the second author began with an appropriately normalized set of transforms (under modular substitutions) of $u_{3}(\tau):=$ $\eta(\tau / 3) / \eta(\tau)$. However, he eventually realized that the modular equations obtained for these 'generalized Weber functions' did not appear to vary as $n$ increased. Moreover, the single identity that he found was completely general in that the second parameter $n \tau$ was not related to $\tau$ in any way, i.e., the equation held for two completely independent complex variables. Simplification then gave the identity for the eta-function given in Theorem 1.1 above. The identity was then verified in many cases to tens of thousands of decimal places.

\section{Proof of Theorem 1.1}

Let $q=e^{2 \pi i w}, Q=e^{2 \pi i z}$, and $\rho=e^{2 \pi i / 3}$. Then (1.1) is equivalent to the identity

$$
\begin{aligned}
27 q^{3 / 8} Q^{3 / 8}\left(q^{3} ; q^{3}\right)_{\infty}^{3}\left(Q^{3} ; Q^{3}\right)_{\infty}^{3}=q^{1 / 24} Q^{1 / 24}\left(q^{1 / 3} ; q^{1 / 3}\right)_{\infty}^{3}\left(Q^{1 / 3} ; Q^{1 / 3}\right)_{\infty}^{3} \\
+i \rho^{1 / 4} q^{1 / 24} Q^{1 / 24}\left(\rho q^{1 / 3} ; \rho q^{1 / 3}\right)_{\infty}^{3}\left(\rho Q^{1 / 3} ; \rho Q^{1 / 3}\right)_{\infty}^{3} \\
-i \rho^{-1 / 4} q^{1 / 24} Q^{1 / 24}\left(\rho^{-1} q^{1 / 3} ; \rho^{-1} q^{1 / 3}\right)_{\infty}^{3}\left(\rho^{-1} Q^{1 / 3} ; \rho^{-1} Q^{1 / 3}\right)_{\infty}^{3}
\end{aligned}
$$

or

$$
\begin{aligned}
27 q^{1 / 3} Q^{1 / 3}\left(q^{3} ; q^{3}\right)_{\infty}^{3}\left(Q^{3} ; Q^{3}\right)_{\infty}^{3}= & \left(q^{1 / 3} ; q^{1 / 3}\right)_{\infty}^{3}\left(Q^{1 / 3} ; Q^{1 / 3}\right)_{\infty}^{3} \\
& +i \rho^{1 / 4}\left(\rho q^{1 / 3} ; \rho q^{1 / 3}\right)_{\infty}^{3}\left(\rho Q^{1 / 3} ; \rho Q^{1 / 3}\right)_{\infty}^{3} \\
& -i \rho^{-1 / 4}\left(\rho^{-1} q^{1 / 3} ; \rho^{-1} q^{1 / 3}\right)_{\infty}^{3}\left(\rho^{-1} Q^{1 / 3} ; \rho^{-1} Q^{1 / 3}\right)_{\infty}^{3}
\end{aligned}
$$

To prove (2.1), we use Jacobi's identity [1, p. 285]

$$
(q ; q)_{\infty}^{3}=\sum_{n=0}^{\infty}(-1)^{n}(2 n+1) q^{n(n+1) / 2} .
$$

Observe that

$$
\rho^{n(n+1) / 2}= \begin{cases}1, & \text { if } n \equiv 0,2(\bmod 3) \\ \rho, & \text { if } n \equiv 1(\bmod 3) .\end{cases}
$$

Hence, by (2.2),

$$
\begin{aligned}
\left(\rho q^{1 / 3} ; \rho q^{1 / 3}\right)_{\infty}^{3} & =\sum_{n=0}^{\infty}(-1)^{n}(2 n+1) \rho^{n(n+1) / 2} q^{n(n+1) / 6} \\
& =\sum_{\substack{n=0 \\
n \equiv 0,2(\bmod 3)}}^{\infty}(-1)^{n}(2 n+1) q^{n(n+1) / 6}+\rho \sum_{\substack{n=0 \\
n \equiv 1(\bmod 3)}}^{\infty}(-1)^{n}(2 n+1) q^{n(n+1) / 6}
\end{aligned}
$$




$$
\begin{aligned}
& =\left(q^{1 / 3} ; q^{1 / 3}\right)_{\infty}^{3}+(\rho-1) \sum_{n=0}^{\infty}(-1)^{3 n+1}(6 n+3) q^{(3 n+1)(3 n+2) / 6} \\
& =\left(q^{1 / 3} ; q^{1 / 3}\right)_{\infty}^{3}-3(\rho-1) q^{1 / 3} \sum_{n=0}^{\infty}(-1)^{n}(2 n+1) q^{3 n(n+1) / 2} \\
& =\left(q^{1 / 3} ; q^{1 / 3}\right)_{\infty}^{3}-3(\rho-1) q^{1 / 3}\left(q^{3} ; q^{3}\right)_{\infty}^{3},
\end{aligned}
$$

where we used (2.2) twice again. For brevity, set

$$
A:=\left(q^{1 / 3} ; q^{1 / 3}\right)_{\infty}^{3}, \quad B:=\left(Q^{1 / 3} ; Q^{1 / 3}\right)_{\infty}^{3}, \quad C:=q^{1 / 3}\left(q^{3} ; q^{3}\right)_{\infty}^{3}, \quad D:=Q^{1 / 3}\left(Q^{3} ; Q^{3}\right)_{\infty}^{3}
$$

Using the notation above, (2.3), its analogue with $\rho$ replaced by $\rho^{-1}$, and their analogues, with $q$ replaced by $Q$, in (2.1), we find that it suffices to prove that

$$
\begin{aligned}
27 C D= & A B+i \rho^{1 / 4}(A-3(\rho-1) C)(B-3(\rho-1) D) \\
& -i \rho^{-1 / 4}\left(A-3\left(\rho^{-1}-1\right) C\right)\left(B-3\left(\rho^{-1}-1\right) D\right) .
\end{aligned}
$$

Observe that $\rho^{1 / 4}=(\sqrt{3}+i) / 2$. Thus, the coefficient of $A B$ on the right-hand side of (2.4) is equal to

$$
1+i \rho^{1 / 4}-i \rho^{-1 / 4}=1+i\left(\frac{\sqrt{3}}{2}+\frac{i}{2}\right)-i\left(\frac{\sqrt{3}}{2}-\frac{i}{2}\right)=0 .
$$

Next, the coefficients of $A D$ and $B C$ on the right-hand side of (2.4) are each equal to

$$
\begin{aligned}
& -3 i \rho^{1 / 4}(\rho-1)+3 i \rho^{-1 / 4}\left(\rho^{-1}-1\right) \\
& =-3 i\left(\frac{\sqrt{3}}{2}+\frac{i}{2}\right)\left(\frac{-3+i \sqrt{3}}{2}\right)+3 i\left(\frac{\sqrt{3}}{2}-\frac{i}{2}\right)\left(\frac{-3-i \sqrt{3}}{2}\right) \\
& =-\frac{3 i}{4}(-\sqrt{3})+\frac{3 i}{4}(-\sqrt{3})=0 .
\end{aligned}
$$

The coefficient of $C D$ on the right-hand side of (2.4) is equal to

$$
\begin{aligned}
& 9 i \rho^{1 / 4}(\rho-1)^{2}-9 i \rho^{-1 / 4}\left(\rho^{-1}-1\right)^{2} \\
& =9 i\left(\frac{\sqrt{3}}{2}+\frac{i}{2}\right)\left(\frac{-3+i \sqrt{3}}{2}\right)^{2}-9 i\left(\frac{\sqrt{3}}{2}-\frac{i}{2}\right)\left(\frac{-3-i \sqrt{3}}{2}\right)^{2} \\
& =9 i\left(\frac{6 \sqrt{3}-6 i}{4}-\frac{6 \sqrt{3}+6 i}{4}\right) \\
& =9 i(-3 i)=27 .
\end{aligned}
$$

Hence, using the calculations (2.5)-(2.7) in (2.4), we see that (2.4) indeed has been shown, and so this completes the proof. 


\section{REFERENCES}

[1] G. H. Hardy and E. M. Wright, An Introduction to the Theory of Numbers, 4th ed., Clarendon Press, Oxford, 1960.

[2] W. B. Hart, Evaluation of the Dedekind Eta Function, Ph.D. Thesis, Macquarie University, Sydney, 2004.

[3] H. Weber, Lehrbuch der Algebra, Dritter Band, Friedrich Vieweg und Sohn, Braunschweig, 1908; reprinted by Chelsea, New York, 1961.

Department of Mathematics, University of Illinois, 1409 West Green Street, UrBANA, IL 61801, USA

E-mail address: berndt@math.uiuc.edu

E-mail address: wbhart@math.uiuc.edu 